\title{
On a Blind Fractionally Sampling-Based Carrier Frequency Offset Estimator for Noncircular Transmissions
}

\author{
Philippe Ciblat, Erchin Serpedin, Member, IEEE, and Yan Wang
}

\begin{abstract}
This letter deals with the problem of nondata-aided carrier frequency offset estimation of noncircular modulations transmitted through unknown frequency-selective channels. By exploiting the unconjugated cyclostationary statistics induced by oversampling in the received waveform, an optimized carrier frequency offset estimator is proposed and its asymptotic (large sample) performance analyzed. In order for the proposed frequency estimator to achieve the minimum mean-square estimation error, it is shown that the oversampling rate need not be larger than twice the symbol rate. It is also shown that the proposed synchronizer is asymptotically jitter-free, admits a feedforward structure that may be implemented in digital form, and is suitable for burst transmissions.
\end{abstract}

Index Terms-Frequency-selective channel, nondata-aided estimation, oversampling, synchronization.

\section{INTRODUCTION}

$\mathbf{T}$ HE GROWTH OF mobile wireless communications systems has prompted an increased interest in designing digital receivers operating on samples of the fractionally sampled (oversampled) received signal. The main reason is that oversampling gives rise to cyclostationarity (CS), which implies that more statistical information can be used for designing digital receivers with improved performance.

Several carrier frequency-offset estimators for flat-fading channels that exploit the conjugated second-order cyclostationary statistics of the oversampled received signal were proposed in [1], [3], and [5]. A high-performance blind maximum-likelihood (ML) framework to estimate the Doppler shift in the presence of an unknown frequency selective channel was proposed in [6]. However, the proposed ML-solution relies on an iterative Baum-Welch type algorithm, whose large numerical complexity and possible lack of convergence prohibit its use for many practical applications [6]. Tong [7] proposed a different solution for blind joint detection and carrier recovery in the presence of an unknown multipath channel using a Viterbi-like algorithm. Since this solution relies on a critical

Manuscript received May 20, 2002; revised September 5, 2002. The associate editor coordinating the review of this manuscript and approving it for publication was Prof. Dimitris A. Pados.

P. Ciblat is with the Département Communications et Electronique, Ecole Nationale Supérieure des Télécommunications, 75013 Paris, France (e-mail: ciblat@com.enst.fr).

E. Serpedin and Y. Wang are with Department of Electrical Engineering Texas A\&M University, College Station, TX 77843 USA (e-mail: serpedin@ ee.tamu.edu).

Digital Object Identifier 10.1109/LSP.2003.809031 channel identifiability condition, which may not be always satisfied, the applicability of this algorithm is also limited [7].

The above-mentioned considerations hold irrespective of the nature of the transmit signal constellation: circular or noncircular. This letter shows that the estimation of the carrier frequency offset estimation can be significantly simplified when the constellation is noncircular (e.g., real-valued constellation such as binary phase-shift keying). Under this assumption, this letter proposes a blind frequency offset estimator that does not require knowledge of the multipath channel and transmitted data sequence. The proposed synchronizer admits a feedforward structure that may be easily implemented in digital form, does not present high computational complexity, exhibits much faster convergence rates $\left(O\left(1 / N^{3}\right), N\right.$ denotes the number of available data samples) than the algorithms [1], [3], [5], whose convergence rates are $O(1 / N)$, and its convergence and consistency are guaranteed even in the presence of unknown multipath effects.

In this letter, the frequency offset estimator is designed in an optimized manner by exploiting efficiently all the unconjugated second-order statistics that are present in the received waveform. By performing a rigorous performance analysis, the mse of the resulting carrier estimator is shown to be invariant with respect to the oversampling factor $P$ as soon as $P \geq 2$ and its minimum mse is achieved when $P=2$. Thus, selecting a low oversampling factor (e.g., $P=2$ ) leads to an optimal carrier recovery scheme that requires a low complexity receiver, and without being necessary to adopt large oversampling factors. From this perspective, the present carrier synchronizer represents a generalization of the estimators reported earlier by the authors in [2] and [9], which exploit only a subset of the received signal's unconjugated second-order CS statistics.

We remark also that [8] represents a different extension of the results reported in [2]. Ciblat and Vandendorpe [8] propose a unifying carrier frequency estimation framework that can be used for systems employing linear block precoders at the transmitter. However, only code division multiple access and orthogonal frequency division multiplexing schemes are analyzed within this unifying transmitter precoding setup [8].

Since the operation of oversampling the received waveform may be interpreted as a transmitter precoding scheme, the theoretical asymptotic performance analysis framework in [2] and [8] is exploited herein to design optimized frequency recovery schemes that operate on asynchronous signal samples taken at a rate faster than the symbol rate $\left(T_{s}\right)$. Thus far, to the best knowledge of the authors, such an analysis has not been reported in 
the literature and our goal herein is to complete this analysis by choosing the optimal set of parameters (in particular, number of cyclic correlation lags and oversampling rate) that have to be considered in the frequency offset estimator so that its asymptotic variance is minimized.

\section{PROPOSED ESTIMATOR}

We focus on single-carrier and single-user wireless communications channels. The continuous-time base-band received signal $y_{a}(t)$ can be expressed as follows:

$$
y_{a}(t)=\left(\sum_{k \in \mathbb{Z}} s_{k} h_{a}\left(t-k T_{s}\right)\right) e^{2 i \pi f_{a} t}+w_{a}(t)
$$

where the symbol sequence $\left\{s_{k}\right\}$ transmitted at the baud rate $1 / T_{s}$ is assumed to be noncircular (i.e., $\mathbb{E}\left[s_{k}^{2}\right] \neq 0$ ), independently and identically distributed with zero-mean and unit-variance. The filter $h_{a}(t)$, which is assumed to be time-limited and causal, and results from the convolution of the multipath propagation channel and the shaping filter, supposed of bandwidth $\left(-(1+\rho) / 2 T_{s},(1+\rho) / 2 T_{s}\right)$, with the roll-off factor $\rho \in[0,1]$. The additive noise $w_{a}(t)$ is assumed to be white circularly and normally distributed with power spectral density $2 N_{0}$. Finally, $f_{a}$ stands for the analog carrier frequency offset, which may be induced by the local oscillator drifts and Doppler effects.

In general, the parameters $f_{a}$ and $h_{a}(t)$ are unknown and have to be estimated in order to detect the transmitted data $s_{k}$. This letter proposes an optimized frequency-offset compensator that does not require knowledge of the channel impulse response or training sequence. The proposed frequency estimator is implemented digitally and assumes at the front end of the receiver an antialiasing filter (AAF) $g_{a}(t)$, whose output is sampled at the rate $1 / T=P / T_{s}$, where the oversampling factor $P$ is an integer (e.g., see [4, p. 139]). The AAF is assumed to be an ideal low-pass filter (although less stringent conditions on the frequency response of the AAF may be adopted ) with bandwidth $B$ sufficiently large in order to preserve all the signal components at the filter output. The output of the AAF, denoted by $z_{a}(t)$, is sampled so that Nyquist's condition is satisfied $1 / T=2 B$ (i.e., $\left.B=P / 2 T_{s}\right)$. Since $z_{a}(t):=g_{a}(t) * y_{a}(t)$, where $*$ stands for the convolution operation, the following discrete-time channel model is obtained:

$$
z(k):=z_{a}(k T)=\left(\sum_{m=0}^{L} l_{m} v_{k-m}\right) e^{2 i \pi f_{d} k}+n(k)
$$

where $\left\{v_{k}\right\}$ stands for the sequence obtained by padding $P-1$ zeros between any two consecutive symbols $\left\{s_{k}\right\}$. Define also $l(z):=\sum_{m=0}^{L} l_{m} z^{-m}$, with $l_{m}:=\left.\left(g_{a}(t) * h_{a}(t)\right)\right|_{t=m T}$, the digital frequency offset $f_{d}:=f_{a} T$, and the discrete-time white noise sequence $n(k):=\left.\left(g_{a}(t) * w_{a}(t)\right)\right|_{t=k T}$ of variance $\sigma^{2}:=\mathbb{E}\left[|n(k)|^{2}\right]=2 N_{0} / T$. Our goal is to build an optimal frequency offset estimator based on the sole knowledge of the observation samples $\{z(k)\}_{k=0}^{N-1}$. In essence, we propose an estimator that optimally exploits all the unconjugated second-order cyclic statistics of the received data and show that its asymptotic variance is invariant with respect to the oversampling factor $P$ as soon as $P \geq 2$.
Since the channel model (1) works as if one has transmitted the block sequence $\left[v_{k P}, \ldots, v_{k P+P-1}\right]=\mathbf{k}^{T} s_{n}$, with $\mathbf{k}=\left[1, \mathbf{0}_{1, P-1}\right]^{T}$, (1) can be interpreted as a linearly precoded system. Consequently, we can use the frequency offset estimator [8] that holds for general linear precoders, described by a tall matrix $\mathbf{K}$. Let $r_{c}(n, \tau):=\mathbb{E}[z(n+\tau) z(n)]$ denote the unconjugated correlation at lag $\tau$ of $z(n)$, and define $\alpha_{0}:=\left(2 f_{d}\right.$ modulo 1$)$. Considering a Fourier series expansion of $r_{c}(n, \tau)$, we obtain

$$
r_{c}(n, \tau)=\sum_{p=0}^{P-1} r_{c}^{\left(\alpha_{0}+p / P\right)}(\tau) e^{2 i \pi\left(\alpha_{0}+p / P\right) n}
$$

where $r_{c}^{(\alpha)}(\tau)$ stands for the unconjugated cyclocorrelation at lag $\tau$ and cyclic frequency $\alpha$. Let $\mathcal{A}_{0}$ be a compact set included in $[0, \min (1 / 2,1 / P)]$. According to $(2)$, we get

$$
\forall \alpha \in \mathcal{A}_{0}, \alpha \neq \alpha_{0}, \forall \tau, \forall p \quad r_{c}^{(\alpha+p / P)}(\tau)=0 .
$$

Then, an asymptotically unbiased and consistent estimate $\hat{\alpha}_{N}$ of $\alpha_{0}$ is obtained as follows:

$$
\hat{\alpha}_{N}:=\arg \max _{\alpha \in \mathcal{A}_{0}} J_{N}(\alpha) \quad J_{N}(\alpha):=\sum_{p=0}^{P-1}\left\|\hat{\mathbf{r}}_{c, N}^{(\alpha+p / P)}\right\|_{\mathbf{W}_{p}}^{2}
$$

where $\hat{\mathbf{r}}_{c, N}^{(\alpha)}:=\left[\hat{r}_{c, N}^{(\alpha)}(-M), \ldots, \hat{r}_{c, N}^{(\alpha)}(M)\right]^{T} ; M$ denotes a positive integer $(M \geq L)$; and $\left\{\mathbf{W}_{p}\right\}_{p=0}^{P-1}$ is a sequence of positive-definite Hermitian matrices. (If $\mathbf{x}$ and $\mathbf{W}$ denote a vector and a positive Hermitian matrix, respectively, then by definition $\|\mathbf{x}\|_{\mathbf{W}}^{2}:=\mathbf{x}^{H} \mathbf{W} \mathbf{x}$. Superscripts ${ }^{T}$ and ${ }^{H}$ stand for transposition and complex-conjugate transposition, respectively.) The term $\hat{r}_{c, N}^{(\alpha)}(\tau):=(1 / N) \sum_{n=0}^{N-1} z(n) z(n+\tau) e^{-2 i \pi \alpha n}$ denotes a sample estimate of $r_{c}^{(\alpha)}(\tau)$, assuming $N$ observations available.

A few observations are now in order. The introduced estimate is an extension of the estimator [9], which exploits only one cyclic correlation lag $(M=0)$. Second, the introduced estimator may be interpreted as a special case of the estimators proposed in [8], which are associated with different precoding schemes. Nevertheless, the design and analysis of the new estimator reported herein remain of interest and have not been reported in the literature.

By exploiting the results of [8], the consistency and asymptotic normality of $\hat{\alpha}_{N}$ can be established. In addition, a closed-form expression for the asymptotic covariance defined as

$$
\gamma:=\lim _{N \rightarrow \infty} N^{3} \mathbb{E}\left[\left(\hat{\alpha}_{N}-\alpha_{0}\right)^{2}\right]
$$

may be obtained. Furthermore, in order to minimize the asymptotic variance $\gamma$, it can be shown that it is optimal to consider $M=L$ and $\mathbf{W}_{p}=\delta_{0, p} \mathbf{I}_{2 L+1}$, where $\delta$ and $\mathbf{I}_{2 L+1}$ denote the Kronecker index and the $(2 L+1) \times(2 L+1)$-identity matrix, respectively [8]. Simply stated, the extraction of the frequency offset should be performed based solely on the harmonic $\alpha_{0}+p / P$ with $p=0$, and all the cyclic correlation lags have to be taken into account. These results may be derived using similar techniques as the ones presented in [2] and [8], and will not be detailed herein. In the sequel, we focus on the estimate 
associated with such an optimal design setting and analyze its asymptotic performance.

\section{INFLUENCE OF THE OVERSAMPLING FACTOR}

As $M=L$ and $\mathbf{W}_{p}=\delta_{0, p} \mathbf{I}_{2 L+1}$, the closed-form expression of $\gamma$ given in [8] can be reduced to

$$
\gamma=\frac{3 P \sigma^{2}}{\pi^{2} a^{2}}\left(P \sigma^{2} a+2 b\right)
$$

with

$$
a:=\int_{-1 / 2}^{1 / 2}\left|l\left(e^{2 i \pi f}\right)\right|^{2}\left|l\left(e^{-2 i \pi f}\right)\right|^{2} d f
$$

and

$$
b:=\int_{-1 / 2}^{1 / 2}\left|l\left(e^{2 i \pi f}\right)\right|^{4}\left|l\left(e^{-2 i \pi f}\right)\right|^{2} d f .
$$

To properly study the influence of the oversampling factor $P$ on the performance of frequency estimator $\hat{\alpha}_{N}$, we evaluate the following term:

$$
E_{P}=\mathbb{E}\left[\left(f_{a} T_{s}-\left.\widehat{f_{a} T_{s}}\right|_{P, N_{s}}\right)^{2}\right]
$$

with ${\widehat{f_{a} T_{s}}}_{P, N_{s}}:=\hat{\alpha}_{N} P / 2$ and $N_{s}:=N / P$. In fact, $E_{P}$ represents the theoretical mse of the analog frequency offset estimate normalized with the symbol duration, assuming that the duration of the observation window is $N_{s} T_{s}$. From (3) and (4), it turns out that

$$
E_{P}=\frac{\gamma}{4 P N_{s}^{3}}
$$

After some quite straightforward but very long calculations based on Poisson's formulae, (5) can be expressed as

$$
E_{P}=\frac{3 N_{0}}{\pi^{2} N_{s}^{3} \zeta_{P}^{(1)^{2}}}\left(N_{0} T_{s} \zeta_{P}^{(1)}+\zeta_{P}^{(2)}\right)
$$

with

$$
\left\{\begin{array}{ll}
\zeta_{1}^{(1)} & :=\int_{-1 / 2 T_{s}}^{1 / 2 T_{s}}\left|H_{a}(f)\right|^{2}\left|H_{a}(-f)\right|^{2} d f \\
\zeta_{1}^{(2)} & :=\int_{-1 / 2 T_{s}}^{1 / 2 T_{s}}\left|H_{a}(f)\right|^{4}\left|H_{a}(-f)\right|^{2} d f
\end{array} \text { for } P=1\right.
$$

and

$$
\left\{\begin{array}{ll}
\zeta_{P}^{(1)} & :=\int_{-1 / T_{s}}^{1 / T_{s}}\left|H_{a}(f)\right|^{2}\left|H_{a}(-f)\right|^{2} d f \\
\zeta_{P}^{(2)} & :=\int_{-1 / T_{s}}^{1 / T_{s}}\left|H_{a}(f)\right|^{4}\left|H_{a}(-f)\right|^{2} d f
\end{array} \text { for } P \geq 2 .\right.
$$

As soon as $P \geq 2, \zeta_{P}^{(1)}$, and $\zeta_{P}^{(2)}$ do not depend on $P$. Thus, the theoretical mse is independent of $P$, a result which intuitively might be predicted based on Shannon's interpolation theorem, and is equal to zero in the noiseless case $\left(N_{0}=0\right)$ (i.e., an asymptotically jitter-free recovery scheme).

\section{SimULATIONS}

The SNR is expressed regardless of the oversampling factor as SNR $:=\int_{\mathbb{R}}\left|H_{a}(f)\right|^{2} d f / 2 N_{0}$. We fix also $\rho=0.2, f_{d}=$ $0.05, T_{s}=3 \mu \mathrm{s}$, and the circularly distributed noise $n(k)$ is assumed white and Gaussian. The theoretical and experimental mse of the frequency estimator are obtained by averaging $E_{P}$ and $\left\|f_{a} T_{s}-\left.\widehat{f_{a} T_{s}}\right|_{P, N_{s}}\right\|^{2}$ over MC $=100$ Monte Carlo trials,

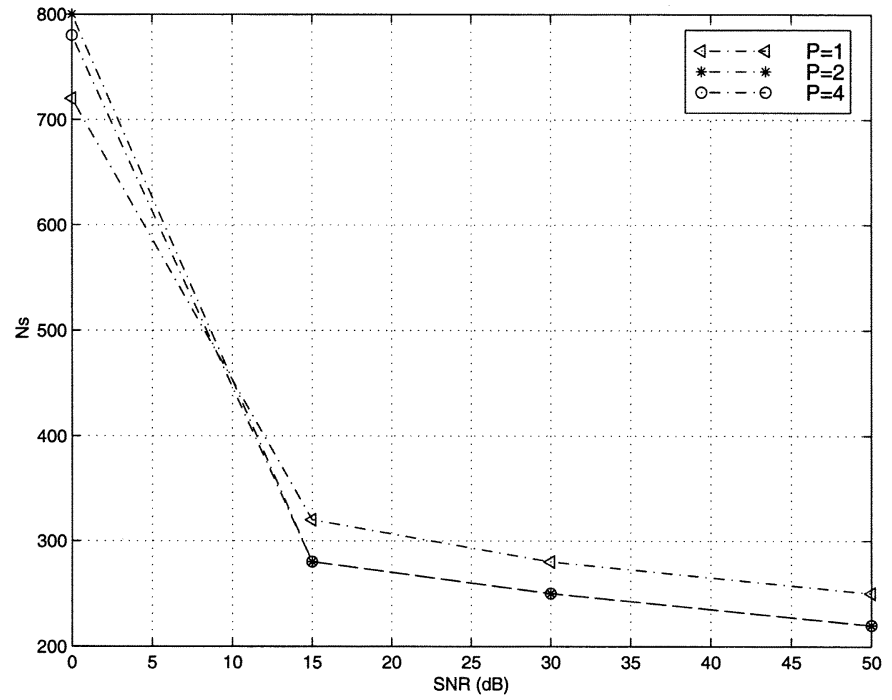

Fig. 1. Lower bound for $N_{s}$ versus SNR.

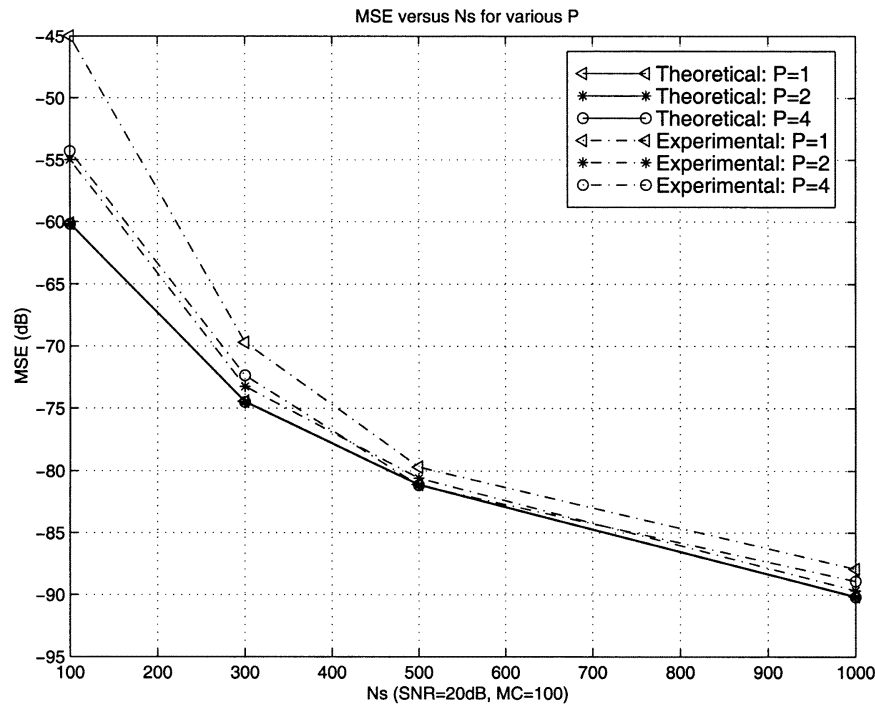

Fig. 2. Theoretical and experimental mse versus $N_{s}$.

respectively. At each trial, a (slow Rayleigh) fading multipath propagation channel with three paths is adopted. The complex amplitudes of the paths are normally distributed and the timing delays assume uniform distributions in $\left[0,3 T_{s}\right]$. As it is usually performed, we proceed in two steps to obtain the frequency offset estimate: first, a coarse search step is performed to maximize the criterion $J_{N}(\alpha)$ via a fast Fourier transform (FFT). Then, a fine search step is performed based on a gradient algorithm, initialized with the estimate provided by the coarse search step. Since the asymptotic analysis studies the behavior of the criterion around the true point $\alpha_{0}$, this analysis does not provide any relevant information on the performance of the first step, which optimizes the criterion over an FFT grid of frequencies spread on the entire interval $[0,1 / P]$. The performance of the first step can be relevantly evaluated by means of the occurrence probability of a wrong detection of the peak, which normally should occur around $\alpha_{0}$. Fig. 1 depicts the number of samples $N_{s}$ with respect to SNR for which the probability of failure is less than $1 \%$. In fact, the amplitude of the spectral line 


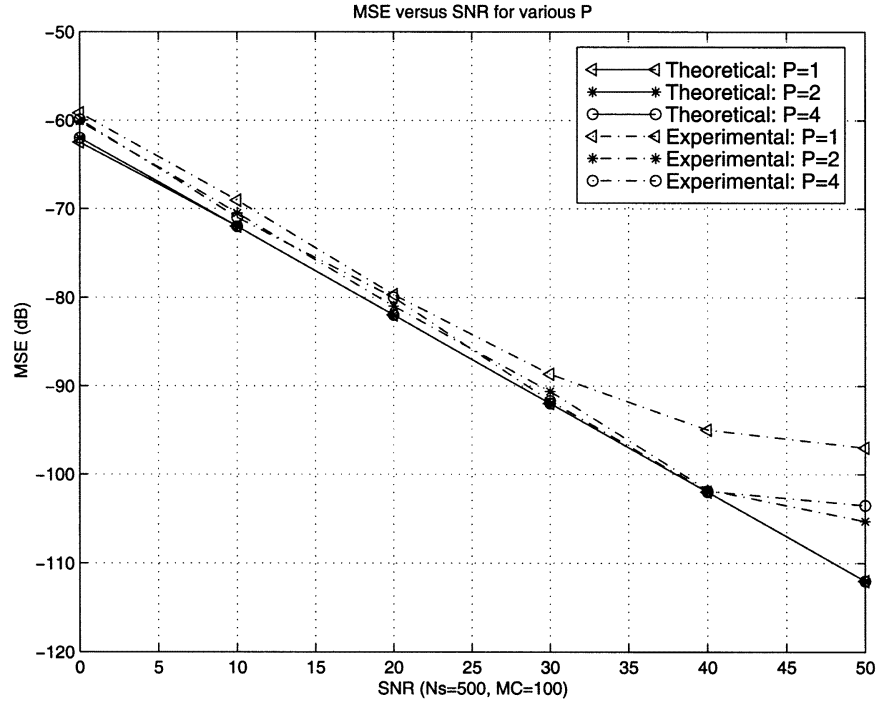

Fig. 3. Theoretical and experimental mse versus SNR.

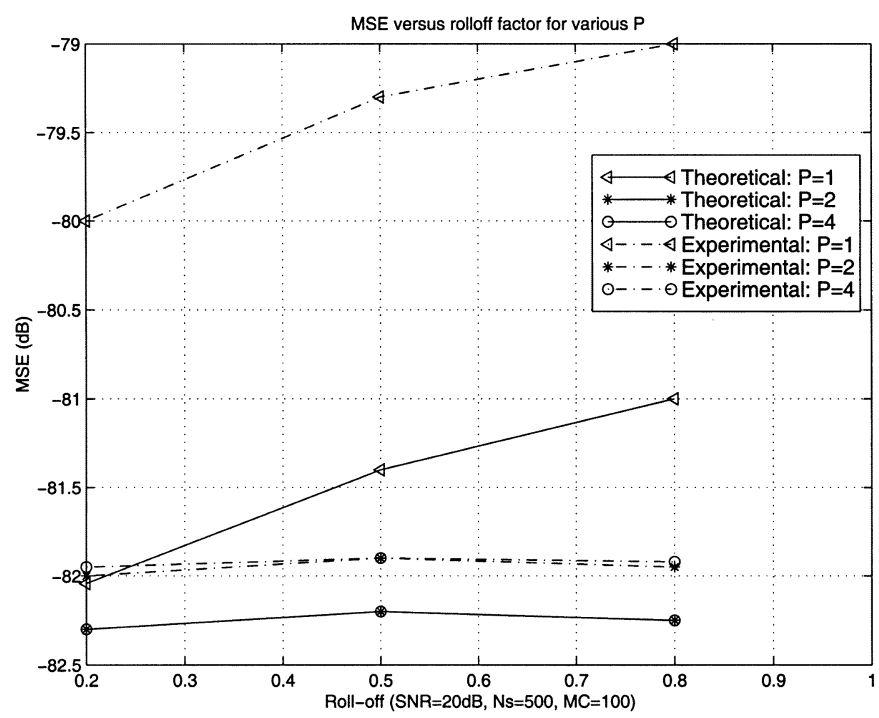

Fig. 4. Theoretical and experimental mse versus $\rho$.

localized at frequency $\alpha_{0}$ depends on the terms $\zeta_{P}^{(k)}, k=1,2$. We have observed that the false detection occurs whenever the terms $\zeta_{P}^{(k)}, k=1,2$, are numerically weak, in general smaller than the noise variance.

In the sequel, we only consider the trials which succeeded to detect the right peak. Fig. 2 plots the mse versus $N_{s}$. We observe that the mse is proportional to $O\left(1 / N_{s}^{3}\right)$. For the remaining sim- ulations, we fix $N_{s}=500$. Fig. 3 plots the mse versus SNR, and shows that the performance of the baud-rate estimator is worse than the performance of the oversampled estimator. In Fig. 4, we depict the mse versus $\rho$. For $P \geq 2$, the performance is quite the same with respect to $\rho$. On the contrary, for $P=1$, the theoretical and experimental performances slightly degrade as $\rho$ increases. Indeed, as the roll-off factor increases, the loss of information becomes more important. Judicious exploitation of the entire statistical information requires to select $P>1$. In all the figures, one can observe that the performance is independent of the oversampling factor $P$ as soon as $P>2$. In addition, the estimation performance corresponding to the oversampled case $(P \geq 2)$ is always better than the baud-rate case $(P=1)$.

\section{CONCLUSION}

We have investigated the theoretical and experimental mse performances of a blind frequency offset estimator based on the unconjugated cyclostationary statistics of the oversampled received signal. In perfect agreement with Shannon's interpolation theorem, we deduced that for optimum performance the oversampling factor does not need to be larger than two. Consequently, receivers with reduced sampling rates (complexity) may be designed without any loss in performance.

\section{REFERENCES}

[1] F. Gini and G. B. Giannakis, "Frequency offset and symbol timing recovery in flat-fading channels: A cyclostationary approach," IEEE Trans. Commun., vol. 46, pp. 400-411, Mar. 1998.

[2] P. Ciblat et al., "Performance analysis of blind carrier frequency offset estimators for noncircular transmissions through frequency-selective channels," IEEE Trans. Signal Processing, vol. 50, pp. 130-140, Jan. 2002.

[3] M. Ghogho, A. Swami, and T. Durrani, "On blind carrier recovery in time-selective fading channels," in Proc. 33rd Asilomar Conf. on Signals, Systems and Computers, vol. 1, 1999, pp. 243-247.

[4] U. Mengali and A. N. D'Andrea, Synchronization Techniques for Digital Receivers. New York: Plenum, 1997.

[5] K. E. Scott and E. B. Olasz, "Simultaneous clock phase and frequency offset estimation," IEEE Trans. Commun., vol. 43, pp. 2263-2270, July 1995

[6] H. A. Cirpan and M. K. Tsatsanis, "Maximum likelihood blind channel estimation in the presence of Doppler shifts," IEEE Trans. Signal Processing, vol. 47, pp. 1559-1569, June 1999.

[7] L. Tong, "Joint blind signal detection and carrier recovery over fading channel," in Proc. ICASSP, Detroit, MI, 1995, pp. 1205-8.

[8] P. Ciblat and L. Vandendorpe, "Blind carrier frequency offset estimation for noncircular constellation based transmission," IEEE Trans. Signal Processing, vol. 51, May 2003, to be published.

[9] Y. Wang, E. Serpedin, P. Ciblat, and P. Loubaton, "Non-data-aided feedforward cyclostationary statistics based carrier frequency offset estimators for linear modulations," in GLOBECOM, vol. 2, San Antonio, TX, Nov. 2001, pp. 1386-1390. 